\title{
Utilization of XANES Imaging in Assessing Radiation Damage in Wheat.
}

$\underline{\text { K.M. Spiers }}^{1 *}$, E. Lemmens ${ }^{2}$, N. De Brier ${ }^{2}$, J.A. Delcour ${ }^{2}$, E. Smolders ${ }^{3}$, J. Garrevoet ${ }^{1}$, G. Falkenberg ${ }^{1}$, G.F. Moorhead ${ }^{4}$ and C.G. Ryan ${ }^{4}$

${ }^{1}$ Photon Science, Deutsches-Elektronen Synchrotron DESY, Hamburg, Germany.

2. Laboratory of Food Chemistry and Biochemistry, Leuven Food Science and Nutrition Research

Centre, KU Leuven, Belgium.

3. Division of Soil and Water Management, KU Leuven, Leuven, Belgium.

4. CSIRO, Clayton, Australia

* Corresponding author, Kathryn.Spiers@desy.de

X-ray fluorescence microscopy (XFM) has been used extensively for examining the chemical elements within grain samples, such as wheat $[1,2]$. Grains can be a major source of micronutrients such as iron; however, only $5-25 \%$ of the total $\mathrm{Fe}$ in wheat is bioavailable for absorption by humans [3], likely due to chelation by phytates [4]. X-ray absorption near edge structure (XANES) spectroscopic imaging can identify the iron species, and thenceforth the processes that can affect the elemental bioavailability. Radiation damage due to exposure to ionizing radiation is a limiting factor in X-ray analysis of samples [5]. It is well known that cryogenic temperatures greatly reduce such damage [6]; however, such measurements can be logistically challenging to perform. STXM XANES has previously been used at room temperature to measure radiation-induced chemical changes to a uniform polymer film sample (PMMA), enabling prediction of bond behaviour under further radiation conditions [7]. The study presented here applies XANES imaging in fluorescence mode on a non-uniform sample, namely a cross section of winter wheat (Cellule, harvest 2015), to spatially identify radiation changes.

The data were collected on the P06 microprobe endstation at PETRA III at DESY. The beam was focused to a spot of $0.97 \times 0.90 \mu \mathrm{m}$ to approximate the selected pixel size of $1 \mu \mathrm{m}$ to best ensure uniform illumination of the sample. The fluorescence signal was collected using the Maia 384C detector in backscatter geometry. XANES imaging was performed at 77 energies from 7.062 to $7.250 \mathrm{keV}$ across the Fe K-edge (7.112 keV). All Maia data were analyzed with the GeoPIXE v7.4i package [8, 9].

An XRF map of the scanned area of the wheat grain is shown in Figure 1. This area was scanned repeatedly to accumulated 6 XANES stacks, with 77 individual 2D maps across the Fe K-edge. The exposure time used for each stack, and the corresponding exposure time accumulated by this area of the wheat grain is shown in Table 1. Three different regions have been identified in the scanned area; aleurone tissue, the main storage organ of iron in wheat, an area of modified aleurone and vascular bundle, which is involved in transport of iron to the aleurone, and part of the nucellar projection, which is the tissue where iron enters the grain. XANES spectra were extracted for the total scan area, giving a "bulk" measurement (Figure 2A). It can be seen that with increasing exposure time, the energy of the edge position is decreasing. Similarly, XANES spectra were extracted for each of the three tissue areas identified; for clarity, only the spectra corresponding to stack 1 ( $0.1 \mathrm{~ms}$ exposure time) and stack 6 (1.6 ms exposure time, $3.2 \mathrm{~ms}$ accumulated exposure time) are shown (Figure 2B). The respective "bulk" spectra enable comparison to illustrate the average shift. It can be seen from the pairs of spectra that each area consists of different iron species; however, these species demonstrate different responses to radiation exposure. The aleurone (area 1) shows the largest edge shift, along with changing spectral shape. The area of modified aleurone and vascular bundle (area 2) shows a slight edge shift, possible 
related to the aleurone in this region. The nucellar projection (area 3) appears to demonstrate no spectral change.

This study demonstrats that XANES imaging may be applied as a tool for assessment of the susceptibility to radiation damage of spatially-distinct regions of XANES target-ion compounds. This application may extend to assessing whether cryogenic conditions are required for particular X-ray speciation studies.

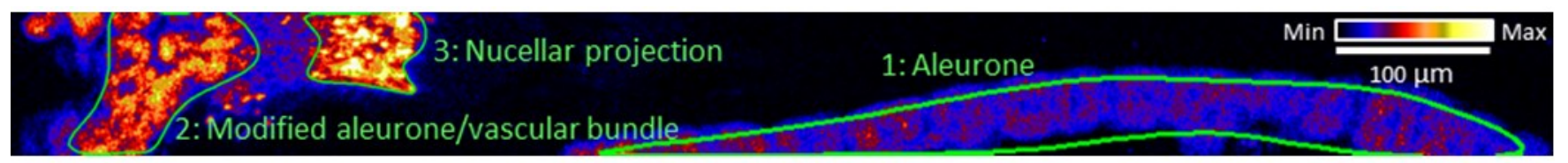

Figure 1: Scanned area within the crease of the wheat grain, highlighting 3 regions: (1) Aleurone tissue (2) Modified aleurone/vascular bundle (3) Nucellar projection.

Table 1: Individual XANES stack exposure time, and accumulated exposure times for the 6 XANES stacks collected on the area of wheat grain shown in Figure 1.

\begin{tabular}{|c|c|c|}
\hline $\begin{array}{c}\text { XANES } \\
\text { Stack }\end{array}$ & $\begin{array}{c}\text { Stack Exposure } \\
(\mathrm{ms})\end{array}$ & $\begin{array}{c}\text { Accumulated } \\
\text { Exposure }(\mathrm{ms})\end{array}$ \\
\hline 1 & 0.1 & 0.1 \\
\hline 2 & 0.1 & 0.2 \\
\hline 3 & 0.2 & 0.4 \\
\hline 4 & 0.4 & 0.8 \\
\hline 5 & 0.8 & 1.6 \\
\hline 6 & 1.6 & 3.2 \\
\hline
\end{tabular}

Figure 2: (A) "Bulk" XANES of the 6 XANES stacks showing average reductive shift. (B) XANES of stack 1 and 6 , for 3 regions shown in Figure 1.

References:

[1] E. Lombi et al, New Phyt. 184 (2009) 193.

[2] E. Lombi et al, J. Exp. Biol. 62 (2010) 273.

[3] N. De Brier et al, J. Agric Food Chem. 63 (2015) 1276.

[4] E. Lemmens et al, Food Chem. (2018) 10.1016/j.foodchem.2018.04.125

[5] T. Beetz and C Jacobsen, J. Synch. Rad. 10 (2002) 280.

[6] R. M. Glaeser and K. A. Taylor, J. Microsc., 112 (1977) 127.

[7] X. Zhang et al, J. Vac. Sci. Technol. 13(4) (1995) 1477.

[8] R. Kirkham et al, AIP Conf. Proc. 1234 (2010) 240.

[9] C. G. Ryan et al, Nucl. Instr. Meth. A 619 (2010) 37.

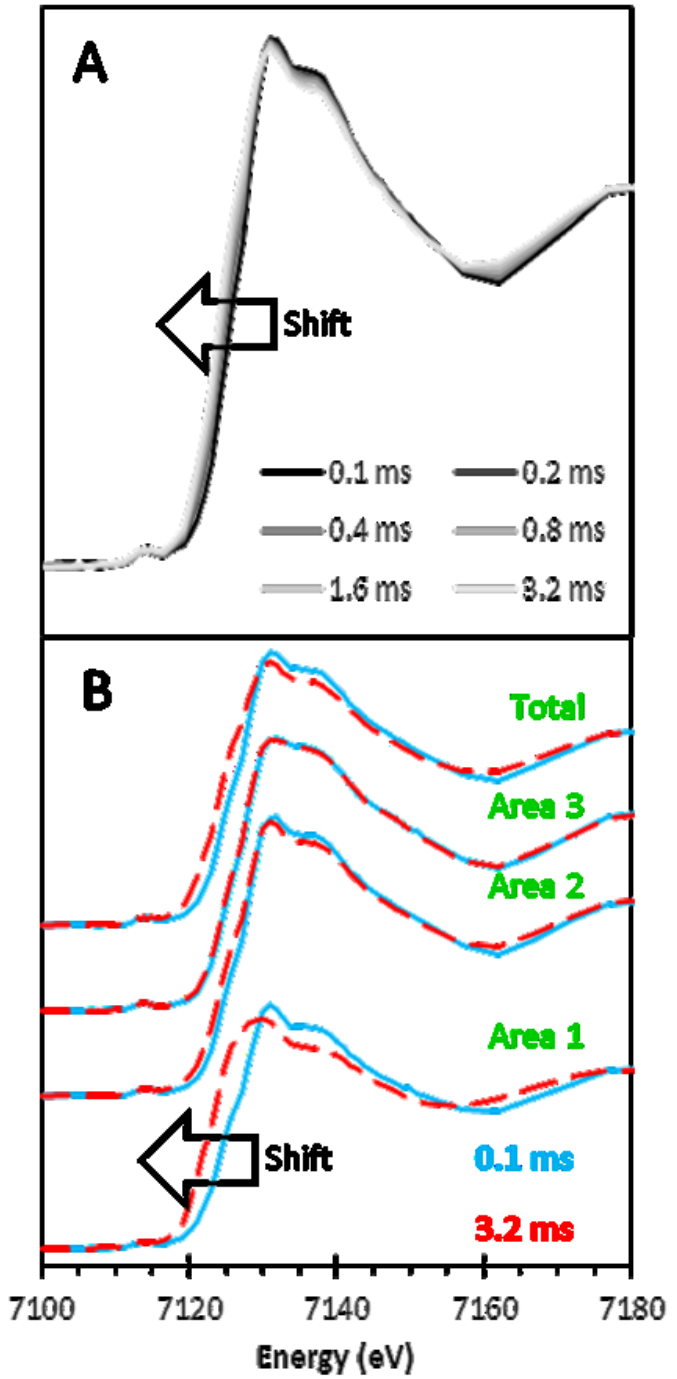

Parts of this research were carried out at P06, PETRA III at DESY, a member of the Helmholtz Association (HGF). All figures and pictures by the author(s) under a CC BY license. 